\title{
Enseñanza virtual en tiempos de emergencias: continuidades y transformaciones
}

\author{
Eduardo Rodríguez Sanabia \\ Natalia Moreira \\ Romina Hortegano \\ Universidad de la República (UdelaR), Uruguay
}

Resumen. Con la pandemia por la COVID-19, la virtualidad se instaló en nuestras vidas ofreciendo una alternativa para sostener actividades y relaciones, incluyendo las prácticas de enseñanza universitaria. En tal contexto, este estudio se propuso analizar las prácticas de enseñanza de una institución de educación superior, a partir de las respuestas y adecuaciones realizadas por los equipos docentes, dando cuenta de las transformaciones, las continuidades y los nuevos desafíos que implican las nuevas realidades educativas y sociales. Utilizamos la sistematización de experiencias, no sólo organizando información que se generó desde el inicio de la pandemia, sino poniendo tales insumos en diálogo con otros nudos críticos que muestran el devenir de la experiencia y que se sometieron a valoración con diversos actores; para lograrlo, se utiliza la metodología narrativa recuperando testimonios, inquietudes y reflexiones por parte del colectivo docente. Como resultado se pudieron visibilizar los aspectos más relevantes dentro de un momento en que los cambios en las prácticas de enseñanza no sólo deben organizarse para atender la eventualidad pandémica, sino que deben reflexionarse de modo crítico en atención al modelo de universidad y de sociedad que se quiere edificar en Uruguay hoy.

Palabras clave: Enseñanza virtual; sistematización de experiencias; docencia universitaria.

\section{Ensino virtual em tempos de emergências: continuidades e transformações}

Resumo. Com a pandemia do Covid-19 a virtualidade instalou-se nas nossas vidas oferecendo uma alternativa para manter atividades e relações, incluindo as práticas de ensino universitário. Nesse contexto, este estudo se propôs a analisar as práticas de ensino de uma instituição de educação superior, a partir das respostas e adequações realizadas pelas equipes docentes, apresentando as transformações, as continuidades e os novos desafios que envolvem as novas realidades educativas e sociais. Utilizamos a sistematização de experiências, não somente organizando a informação gerada desde o início da pandemia, mas também lançando esses componentes ao diálogo com outros pontos críticos que mostram a realidade da experiência e que foram submetidos a avaliação com diversos agentes; para conseguir esse resultado, utiliza-se a metodologia narrativa recuperando depoimentos, inquietudes e reflexões por parte dos docentes. Como resultado foi possível visibilizar os aspectos mais relevantes dentro de um momento no qual as mudanças nas práticas de ensino não só devem ser organizadas para atender a eventualidade pandêmica, porém devem ser refletidas de modo crítico atendendo o modelo de universidade e de sociedade que se pretende construir no Uruguai atualmente. Palavras-chave: ensino virtual; sistematização de experiências; docência universitária.

\section{Virtual teaching in emergencies' times: continuities and transformations}

Abstract. With the Covid-19 pandemic, virtuality became part of our lives, offering an alternative to sustain activities and relationships, including university teaching practices. In this context, this study set out to analyze the teaching practices of a higher education institution, based on the responses and adjustments made by the teaching teams, taking into account the transformations, continuities and new challenges implied by the new educational and social realities. We used the systematization of experiences, not only organizing information generated since the beginning of the pandemic, but also putting such inputs in dialogue with other critical nodes that show the evolution of the experience and that were subjected to assessment with various actors; to achieve this, the narrative methodology is used, recovering testimonies, concerns and reflections by the teaching staff. As a result, the most relevant aspects were made visible at a time when changes in teaching practices should not only be organized to meet the pandemic eventuality, but should also be critically reflected upon in relation to the model of university and society to be built in Uruguay today.

Keywords: virtual teaching; systematization of experiences; universitary teaching. 


\section{Introducción}

En los contextos universitarios, tras la aparición de la pandemia, lo que se manifestó inicialmente fue la preocupación por la continuidad de los procesos académicos, con especial énfasis en la garantía al derecho a la educación por parte del estudiantado. La virtualidad devino parte de la vida cotidiana y de la realidad, y fue necesario operar modificaciones drásticas en todas las prácticas educativas. En palabras de Dussel, Ferrante y Pulfer (2020):

Han sido, son, tiempos extraños. Inicialmente se produjo una especie de hiato, de interrupción abrupta de lo cotidiano, que prometía ser corta. Comenzó un tiempo de espera: de las noticias, de la recuperación del espacio público, de la vacuna, de los avances en la búsqueda de una cura. El tiempo de la pandemia parece de a ratos detenido, lento, pero también acelerado, intensificado (...). Es una experiencia del límite: está claro que no volveremos a ser los mismos (p.11)

La incorporación de la enseñanza virtual a través de diversos medios y recursos en el contexto universitario no sólo implicó modificaciones azarosas de cursos, seminarios y unidades curriculares, sino que también convivió con otra serie de factores vinculantes que le imprimieron condiciones peculiares en cada caso y que otorgaron distintos tonos a la experiencia. Adicionalmente, la velocidad con la que se requerían las respuestas obligó a realizar adecuaciones muy creativas en la mayoría de los casos, con numerosas dificultades las más de las veces.

En medio de estas diatribas, el debate necesario en el contexto educativo pasa por interrogar los potenciales transformadores de las nuevas tecnologías implementadas en educación, en el marco de las diversas experiencias desplegadas para atender las actividades de enseñanza en la universidad. Por ello, partimos de consideraciones teóricas vinculadas con la enseñanza virtual, las competencias digitales docentes y la evaluación de los aprendizajes en la virtualidad, cuyas precisiones resultan vinculantes con este estudio en la Facultad de Humanidades y Ciencias de la Educación (FHCE) de la Universidad de la República (Udelar), Uruguay.

Como parte de esta reflexión, consideramos importante conocer las transformaciones, las continuidades y los nuevos desafíos que componen la panorámica de la enseñanza universitaria en la FHCE. Todo ello luego de la implementación de diversos tipos de herramientas virtuales para sostener el desarrollo de las actividades académicas en el marco de la pandemia, con miras a profundizar la problematización en cuanto a las funciones universitarias y el rol docente hoy.

Con este propósito, se analiza una serie de relevamientos aplicados desde la Unidad de Apoyo a la Enseñanza (UAE) durante el 2020 para obtener información acerca de las diversas medidas y acciones tomadas por el personal docente para desarrollar sus cursos en la FHCE. Asimismo, se consideran todos los aportes y opiniones emitidas por numerosos/as docentes que, en distintas instancias de consultas, relevamientos, clases virtuales, foros en el EVA (Entorno Virtual de Aprendizaje), entre otros, nos hicieron llegar sus impresiones acerca de lo que estaban viviendo durante este proceso. Cabe destacar que, si bien todos los ánimos se centraron en garantizar el derecho del estudiantado, implicó grandes esfuerzos por parte de cada docente para adecuarse a las condiciones del momento y cumplir con tal derecho, a 
expensas de su tiempo, sus habilidades, recursos, situaciones personales y familiares y conocimientos informáticos. En atención a todo lo anterior: se plantean los siguientes objetivos de trabajo.

\section{Objetivo general}

Realizar un análisis de las prácticas de enseñanza en la FHCE, a partir de las diversas respuestas y adecuaciones realizadas por los equipos docentes, dando cuenta de las transformaciones, las continuidades y los nuevos desafíos que implican las nuevas realidades educativas y sociales.

\section{Objetivos específicos}

a) Analizar la situación desde la que partieron los cursos, y las valoraciones docentes en cuanto a las dificultades en el inicio de la educación a distancia

b) Dar cuenta de las transformaciones y adecuaciones realizadas a lo largo de los dos semestres de este año por parte de los equipos docentes

\section{Referencias teóricas}

\subsection{Enseñanza virtual: Tensiones entre lo institucional lo pedagógico}

Autores entre los que se destacan Dron (2018) y Bates (2018) sostienen que la enseñanza virtual se ha construido con base en las prácticas de enseñanza presencial. En este sentido, las metodologías puestas en juego en la enseñanza virtual a menudo reflejan casi con exactitud los cursos tradicionales, sin reparar críticamente en el desarrollo de un nuevo modelo de enseñanza. Lo que hoy se conoce como enseñanza virtual vino a reemplazar un método anterior conocido como enseñanza por correspondencia, que desafió el lugar de la presencialidad en la educación. Este tipo de modelo se centra en un paradigma educativo altamente directivo, donde el sujeto de la educación queda relegado frente al contenido a ser enseñado. Las críticas a este modelo dieron lugar a repensar las formas de enseñanza a distancia, haciendo uso de los avances de la tecnología. Como señala Martel (2004):

En primer lugar, le transfiere un paradigma "instructivo" a la enseñanza contra el paradigma enfocado ampliamente al estudiante. En consecuencia, este medio es dominado por los discursos sobre los enfoques constructivistas, tal como el de las tecnologías educativas. Enseguida, la transferencia de un paradigma impreso (correspondencia) contra los medios de difusión tecnológica (progresivamente soportados de manera numérica): más rápidos, eclécticos, orales y visuales (audio y videocasetes, audio y teleconferencias, computadora, Internet, etc.) y sobre todo multidireccionales (p. 16-17).

Es preciso entonces, repensar las modalidades de enseñanza hegemónicas y que las mismas sirvan de guía para el desarrollo de un nuevo modelo que explote el potencial de las nuevas tecnologías en la educación. Existen en la actualidad diferentes taxonomías que permiten concebir lo que se conoce como enseñanza virtual. Según Porta y Rodés (2020), en nuestro país en general y en el ámbito universitario en particular están presentes y son de uso frecuente al menos tres modalidades. A saber: aula expandida, semipresencial y a distancia. 
La primera, refiere justamente a la extensión de la clase presencial mediante la búsqueda de recursos utilizando tecnologías. En esta modalidad, el aula virtual opera como apoyo, al tiempo que se incrementa el vínculo con el docente y con otros participantes. Por otra parte, la modalidad semipresencial es la que, de forma integrada y complementaria, combina instancias presenciales con virtuales. En la actualidad los modos semipresenciales -también conocidos como blended learning o modelos híbridos- han ganado gran auge y se evidencian implicancias en las formas de enseñanza y aprendizaje, así como también en las metodologías puestas en juego y en los recursos necesarios para su desarrollo (Polanía, 2014). Debates en torno a la calidad en educación superior colocan a los modelos híbridos como una de las respuestas a las mejoras que los sistemas educativos requieren y se pone particular interés en el rol docente, ubicándolo como un mediador en los procesos educativos (Vera, 2008)

Por último, la modalidad a distancia, que coloca exclusivamente la virtualidad como escenario educativo. Independientemente de su puesta en práctica, estas tres modalidades comparten la característica de que deben ser empleadas tomando en cuenta las posibilidades de interacción en su uso, como es el caso de la colaboración e intercambio entre estudiantes y la comunicación y retroalimentación con el docente.

La conceptualización en torno a la enseñanza virtual que aquí se maneja trae a discusión dos enfoques que, dependiendo del punto de vista que se resuelva, obstaculiza o posibilita el desarrollo de la enseñanza virtual. Por un lado, un enfoque institucional y un enfoque pedagógico-didáctico.

Desde el punto de vista institucional, se contempla un variado conjunto de elementos estructurales a tener en cuenta en el desarrollo de la enseñanza virtual. A saber: las reglamentaciones, la planificación, la caracterización de las tecnologías necesarias, la conectividad, entre otros. No obstante, subyacen aspectos de la cultura institucional, tal vez no tan visibles pero que tensionan la organización institucional. Como lo son la experiencia docente con relación a las tecnologías, la carga horaria destinada a la enseñanza, la posibilidad de armar equipos de trabajo, etcétera ( $\mathrm{Hi}-$ dalgo, León y Pavón, 2002).

Desde un enfoque pedagógico, se requiere en principio problematizar el lugar de «docente» y el de "estudiante». Lion (2017) sostiene que, el aprendizaje mediado por las tecnologías digitales, no es automático y tiene características multidimensionales que le permiten desarrollarse a lo largo del tiempo. En enseñanza virtual se aprende más allá de la intervención directa del docente, y éste adquiere un lugar tal vez no tan protagónico como el que ocupa en las aulas tradicionales. En este sentido, el rol docente se reconfigura en el de un facilitador de los contenidos con la intencionalidad de provocar aprendizajes (Fenstermacher, 1989).

Desde esta mirada y teniendo en cuenta el contexto actual de cambios repentinos, las reglamentaciones institucionales en torno a la organización de la enseñanza se ven interpeladas por situaciones contingentes. Esto genera aún más incertidumbre en cada docente que busca más allá de los marcos normativos las guías para orientar sus prácticas, otorgando así la posibilidad de reflexión pedagógica de la tarea educativa que realiza y buscando apoyos desde el punto de vista didáctico. 


\subsection{Competencias digitales docentes}

El concepto de competencias es polisémico y en el campo de las ciencias de la educación siempre ha causado controversias al tratarse de un enfoque que se enfrenta a los paradigmas tradicionales centrados en la trasmisión de contenidos. Las competencias digitales docentes refieren a un conjunto de habilidades y técnicas vinculadas con la solvencia en la utilización de equipos y/o programas informáticos que permitirán el desarrollo del proceso educativo en la indagación, adquisición y en el procesamiento de la información. También se vinculan a este tipo de competencias, elementos de carácter cognitivo, involucrados con la reflexión crítica en el uso de las tecnologías en la enseñanza y componentes didácticos que fundamentan la incorporación de la tecnología en los procesos de enseñanza y aprendizaje (Vólquez y Amador, 2020).

Existe una dimensión de la docencia en lo que refiere a la enseñanza virtual que pone en tensión el trabajo educativo. Se trata de las distancias entre las nuevas formas de socialización que tienen los estudiantes y lo que se ofrece desde el sistema educativo, reconociendo los cambios tecnológicos y en los sujetos de aprendizaje. Esto requiere asumir y problematizar los desafíos del uso de las tecnologías en la enseñanza, apuntando a hacer un uso crítico de éstas, tal como señalan Castañeda, Esteve y Adell (2018) y Cobo (2019).

Sobre las competencias digitales docentes, Morales (2019) señala inicialmente que el trabajo de los profesores ha tomado dimensiones tan complejas que su trabajo no se puede limitar al dominio de una especialidad en particular. Además, la mencionada autora advierte que las competencias docentes van más allá de la trasmisión de un saber concreto. El conocimiento mediado por las tecnologías insume mayor estudio y esfuerzo para poner en práctica el desarrollo de un modelo pedagógico que oriente las prácticas educativas fomentando la capacidad creativa, de cooperación y de innovación.

La necesidad de formar docentes con competencias digitales para una adecuada inserción de las tecnologías en los procesos educativos es cada vez más latente. Los marcos relevados por Morales (2019) evidencian un conjunto de dimensiones que se deben incluir en la formación de profesores. Éstos reflejan un conjunto de competencias que operan como referencias a ser tenidas en cuenta para el diseño de los planes de formación. Aspectos relacionados con lo pedagógico, técnico, ético, legal, así como también cuestiones vinculadas con lo didáctico, como los son las metodologías, las formas de evaluación, la planificación adecuada y las formas de comunicación, dan cuenta de un conjunto de elementos a ser tenidos en cuenta en el desarrollo de las competencias digitales docentes.

Por otra parte, es de reconocer que los estudiantes cada vez más construyen y difunden conocimiento a través de internet y el universo de herramientas presentes lo facilita Scolari (2018), a través del concepto de "alfabetismo transmedia", plantea que los estudiantes son "prosumidores" (productores y consumidores) capaces de generar y producir contenidos de distinto tipo y niveles de complejidad. En ese marco desarrollan "estrategias informales de aprendizaje" que se inscriben en lo que se ha denominado ecologías de aprendizaje (González-Sanmamed, Sangrà, Souto-Seijo y Estévez, 2018). De este modo es casi un "deber" ofrecer distintas vías para el desarrollo 
de la comunicación (aspecto central de cualquier proceso de enseñanza-aprendizaje), en los diversos formatos que ésta puede darse (ya no sólo el escrito, predominante en el mundo universitario) (Porto, 2012).

\subsection{Evaluación de los aprendizajes en la virtualidad}

Dejando de lado que existen diferentes paradigmas educativos que orientan el trabajo educativo, en contextos de transición a la virtualidad, las formas y concepciones en torno a la evaluación de la enseñanza requieren ser puestas en discusión con el propósito de diseñar actividades evaluativas coherentes con el desarrollo del curso, con sus objetivos y con los contenidos a desarrollar. Como punto de partida, se requiere pensar en la evaluación como un proceso continuo en la tarea de enseñanza. Dicho proceso requiere de una planificación cuidadosa que articule los métodos de aprendizaje, de enseñanza y también de evaluación. Al decir de Rodríguez y Rodés (2020, p. 3) "si enseñamos a partir de la memorización no podemos pretender que el estudiante aprenda a realizar un análisis crítico en el momento de ser evaluado y calificado".

Como sostienen Anijovich y Cappelletti (2017) la expresión de la evaluación no puede limitarse a una calificación matemática, se debe contemplar la posibilidad de que el docente coloque a modo evaluativo una retroalimentación o sugerencia. En escenarios de enseñanza virtual, esto cobra gran relevancia y en particular requiere de pensar las formas en las que se produce la comunicación entre docentes y estudiantes a modo de acompañar el proceso educativo de un modo diferente.

Desde este enfoque, se concibe la evaluación de la enseñanza como un proceso sistemático y progresivo que de manera laxa le permite al docente acompañar la evolución de los aprendizajes que se van suscitando en los estudiantes. La evaluación pensada como proceso, opera como instrumento que regula, mide y permite modificar sobre la marcha las prácticas educativas.

Existen un conjunto variado de tipos de evaluación que se ponen en juego a la hora de interpretar los resultados a lo largo de todo el proceso de enseñanza. Rodríguez, Czerwonogora, Verde y Doninalli (2014), señalan los siguientes: evaluación contextual, evaluación del diseño, evaluación formativa, evaluación de resultados. Según los mencionados autores, la primera toma en cuenta el espacio dónde se produce el aprendizaje y posibilita la interpretación de las posibilidades, necesidades e intereses de los estudiantes. Por otra parte, la evaluación de diseño pone énfasis en la planificación propuesta a priori, velando por la coherencia de lo propuesto y vigilando que los contenidos sean adecuados a los objetivos y metodologías. Mientras que, la evaluación procesual se centra en el desarrollo de la instancia de enseñanza y aprendizaje. Este tipo de evaluación analiza las acciones llevadas a cabo en la práctica educativa para alcanzar los objetivos propuestos. Y, por último, la evaluación de los resultados está relacionada con la determinación del cumplimiento de los objetivos trazados. Implica medir los productos obtenidos en el marco del proceso de enseñanza y a partir de ellos reconfigurar las acciones a futuro.

En este sentido, uno de los grandes retos que derivan de la transición a la enseñanza virtual y que se tendría que incluir en la adquisición y desarrollo de competencias digitales docentes, es una visión integrada e integradora de la evaluación a través de 
los diversos Sistemas de Gestión del Aprendizaje, centrados prioritariamente en la evaluación formativa, cooperativa y entre pares. Esto también implica, obviamente, una visión distinta de la práctica de enseñanza en su conjunto, ya que el desarrollo de cada Unidad Curricular, curso o programa, requiere otras posturas para su planificación y para la selección y diseño de los recursos y las actividades a desarrollar.

En el marco de estos retos, el análisis que se realiza en este trabajo pasa por una mirada de las transformaciones, que operaron en las prácticas de enseñanza en el marco de la transición a la virtualidad impulsada por la pandemia. De allí emerge la selección metodológica que describimos a continuación.

\section{Metodología}

En cuanto al enfoque metodológico y el recorrido realizado para ejecutar la investigación, se ha utilizado la sistematización de experiencias como metodología, la cual está circunscrita al paradigma sociocrítico (Coppens y Van de Velde, 2005). Esta práctica además, se corresponde con el enfoque de la educación popular (Jara, 1994), razón por la cual, el estudio tiene carácter eminentemente cualitativo, con predominio de la interpretación crítica de los procesos vividos en la experiencia sometida a estudio.

Para tal efecto, se entiende la sistematización como una "interpretación crítica de una o varias experiencias, que a partir de su ordenamiento y reconstrucción, descubre o explicita la lógica del proceso vivido, los factores que han intervenido en dicho proceso, cómo se han relacionado entre sí, y por qué lo han hecho de ese modo" (Borjas, 2003). Asimismo, la sistematización de experiencias se realiza para producir nuevos conocimientos, que permitirán, a su vez, transformar realidades y prácticas, mediante la reflexión y la acción colectiva.

La sistematización de experiencias no sólo incluye la organización de información, sino el diálogo entre estos datos y los nudos críticos que, convertidos en unidades de análisis, puedan dar cuenta del devenir de la experiencia analizada, sometida a valoración conjuntamente con sus actores. Por ende, se entiende que la realidad de tales experiencias se encuentra en permanente movimiento y que es necesario producir conocimiento sobre nuestras propias prácticas mediante la confluencia entre lo teórico y lo práctico en el devenir de lo vivido y sus reflexiones.

Bajo estas premisas, esta sistematización se organiza a partir de la propuesta metodológica de Barnechea, González y Morgan (1999), dividiendo el plan de trabajo en los siguientes momentos:

1. Unificación de criterios

2. Definición de la imagen-objetivos de la sistematización

3. Reconstrucción de la experiencia

4. Análisis e interpretación de lo sucedido

5. Comunicación.

Es importante destacar que todo lo sistematizado en este trabajo, forma parte de una experiencia que se fue dando como respuesta a las diversas demandas que se produjeron en el contexto de la FHCE y a causa de la pandemia, por ende, las acciones emprendidas y las experiencias que de allí se desprendieron, no fueron 
planificadas con anticipación ni pensadas metodológicamente para una investigación. En consecuencia, el plan de sistematización incluye un proceso de recuperación de lo vivido en función de las diversas situaciones y acciones que fueron teniendo lugar desde nuestra Unidad y con las y los docentes con quienes interactuamos durante el 2020.

Dicho esto, se establecieron como eje para la sistemación, los diversos instrumentos que se utilizaron para recolectar información vinculada con:

1. Intereses formativos antes de la pandemia

2. Relevamiento sobre actividades de enseñanza virtual al inicio de la pandemia

3. Registro situacional de la actividad virtual y su evolución a lo largo del semestre impar

4. Situación de los cursos y actividades de evaluación

Cuadro 1. Secuencia del proceso de investigación. Ejes de sistematización e información sobre el relevamiento.

\begin{tabular}{|c|c|c|c|c|}
\hline \multicolumn{5}{|c|}{ Ejes de sistematización } \\
\hline $\begin{array}{l}\text { Información } \\
\text { sobre el releva- } \\
\text { miento }\end{array}$ & $\begin{array}{l}\text { 1. Intereses for- } \\
\text { mativos antes de } \\
\text { la pandemia }\end{array}$ & $\begin{array}{l}\text { 2. Relevamiento } \\
\text { sobre activida- } \\
\text { des de enseñan- } \\
\text { za virtual al inicio } \\
\text { de la pandemia }\end{array}$ & $\begin{array}{l}\text { 3. Registro si- } \\
\text { tuacional de la } \\
\text { actividad virtual y } \\
\text { su evolución a lo } \\
\text { largo del semes- } \\
\text { tre impar }\end{array}$ & $\begin{array}{l}\text { 4. Situación de } \\
\text { los cursos y } \\
\text { actividades de } \\
\text { evaluación }\end{array}$ \\
\hline $\begin{array}{l}\text { Instrumento de } \\
\text { relevamiento } \\
\text { utilizado }\end{array}$ & $\begin{array}{l}\text { Cuestionario } \\
\text { autoadministra- } \\
\text { do a través de } \\
\text { la plataforma } \\
\text { google }\end{array}$ & $\begin{array}{l}\text { Cuestionario } \\
\text { autoadministra- } \\
\text { do a través de } \\
\text { la plataforma } \\
\text { google }\end{array}$ & $\begin{array}{l}\text { Observación y } \\
\text { registro de cur- } \\
\text { sos en platafor- } \\
\text { ma EVA }\end{array}$ & $\begin{array}{l}\text { Fichas de infor- } \\
\text { mación sobre } \\
\text { equipos docen- } \\
\text { tes, modalidades } \\
\text { de enseñanza y } \\
\text { evaluación }\end{array}$ \\
\hline $\begin{array}{l}\text { Cantidad de } \\
\text { registros }\end{array}$ & 42 docentes & $\begin{array}{l}72 \text { unidades } \\
\text { curriculares }\end{array}$ & 129 cursos & 102 cursos \\
\hline
\end{tabular}

Fuente: elaboración propia

Asimismo, se toman en consideración los aportes de docentes que respondieron los relevamientos y que participaron en las múltiples instancias de consultas, asesorías, orientación, capacitación y formación, especialmente tomando en cuenta que la sistematización "pone en orden conocimientos desordenados y percepciones dispersas que surgieron en el transcurso de la experiencia. Asimismo, explicita intuiciones, intenciones y vivencias acumuladas a lo largo del proceso" (Borjas, 2003, p.16). Es en este punto en el cual se produce una vinculación con la metodología narrativa, ya que permite reconstruir y reorganizar la experiencia a través de los relatos, atribuyendo significados al pasado con referencia al presente y a las concepciones personales. Los sucesos se enmarcan en contextos sociales, culturales e institucionales que les dan sentido y se interpretan en función de la interacción del individuo con el medio (Álvarez, Porta y Sarasa, 2010). 
Con este abordaje se pudieron conocer testimonios de parte de las/os propias/ os docentes quienes nos compartieron sus inquietudes y dificultades, así como sus avances y progresos, con la confluencia de la información y experiencia sistematizadas, y la narrativa de cada docente, se presentan los hallazgos de este trabajo.

\section{Antes, cuando no había pandemia}

La UAE de la FHCE viene transitando desde 2019 un proceso de reestructuración de su equipo docente y sus funciones en procura, entre otras cosas, de fortalecer el desarrollo pedagógico docente. En atención a ello, en octubre del mismo año se realizó un pequeño relevamiento para obtener información relativa a los intereses y necesidades de formación del personal docente en temas vinculados con la enseñanza universitaria y el uso del EVA. Tal instrumento reveló los hallazgos que se describen a continuación:

a) Un 95\% de los/as docentes que respondieron, pertenecen a grados 1, 2 y 3 (31\%, 21\% y 43\%, respectivamente), lo cual consideramos un indicador del interés presente en estos grados por participar en actividades de formación vinculadas con las temáticas antes referidas. Esto viene a ser un potencial de valor en tanto representa la posibilidad de impulsar actividades que contribuyan con la profesionalización y el fortalecimiento de la enseñanza universitaria en sus diversos aspectos. También se vincula con una dinámica concreta relativa al hecho de que son estos grados los que se encargan, entre los grupos docentes, de administrar la plataforma y las actividades y/o recursos que se implementan a través de ella.

b) Entre los temas de mayor interés por parte de las y los docentes que respondieron, se destacan los siguientes:

- Didáctica Universitaria: estrategias innovadoras para la enseñanza de las Humanidades

- Planificación Educativa (Formulación de objetivos y contenidos de enseñanza, planificación de clases y cursos, llenado de formularios de Programas de Unidades Curriculares, vaciado de calificaciones)

- Evaluación Educativa (tipos de evaluación, elaboración de instrumentos, formulación de indicadores de evaluación, relaciones entre evaluación y didáctica)

- Diseño del curso y cargado de material en la plataforma

- Evaluación estudiantil a través de la plataforma (formas de entrega, tareas, consignas, encuestas, parciales, libro de calificaciones)

Tal como podemos observar, estos resultados dan cuenta de un interés más enfocado en aspectos generales de la enseñanza en el contexto universitario, con especial énfasis en la planificación y la evaluación educativa, así como la implementación de esta última a través de EVA. Por otra parte, los temas menos solicitados fueron:

- Enseñanza Universitaria y necesidades educativas especiales

- Uso Educativos de las Redes Sociales

- Las Clases Grabadas 
- Polimedias

- La videoconferencia

- La pizarra interactiva

- El BigBlueButton

- Comunicación a través de la plataforma (mensajería, foros)

Se puede apreciar con estas respuestas, que el uso de los recursos arriba mencionados, no conformaban el universo de necesidades e intereses formativos por parte de las y los docentes que participaron del relevamiento, lo cual es propio de una realidad en la cual las actividades de enseñanza se desarrollaban presencialmente, y no existía un horizonte que apuntara ni siquiera a modalidades mixtas.

Cabe destacar, además, que el uso de EVA no se limita exclusivamente a la virtualidad, sino que puede ser un acompañante de la presencialidad en la medida en que ofrece diversidad de recursos que pueden dinamizar el desarrollo de un curso mediante la incorporación de diversas estrategias de enseñanza y de evaluación. Sin embargo, la impronta de la clase presencial entraña además una forma de trabajo en el aula que caracteriza la enseñanza formal (y más específicamente la universitaria) y cuya trascendencia y transformación ha sido sumamente difícil en nuestras universidades.

Con esta información, la UAE procedió a preparar una oferta de talleres y cursos enfocados a los temas que tuvieron mayor receptividad, incluyendo la problematización del rol del docente en el contexto universitario, especialmente tomando en cuenta el momento que vive la Udelar. Dichas instancias formativas, tendrían inicio para el mes de marzo 2020, sin embargo, nos sorprendió la pandemia y el escenario se transformó drásticamente, pasando a tener prioridad los temas que habían quedado en un plano secundario en el primer relevamiento (El BigBlueButton, videoconferencias, clases grabadas), generando la necesidad de dar respuestas inmediatas y desplegando, por ende, las más diversas instancias de atención para la capacitación rápida y efectiva de docentes

\section{El inicio de la pandemia}

El 13 de marzo de 2020 se confirmaron los primeros casos de Covid-19 en el Uruguay, y a partir de allí se suspendieron las clases presenciales en todos los niveles de enseñanza. En ese momento, desde el Decanato de la FHCE se diseñó un cuestionario autoadministrado a través de la plataforma google para realizar un relevamiento sobre actividades de enseñanza virtual que estaban desarrollando las y los docentes al inicio de la pandemia. El mismo se aplicó entre el 20 de marzo y el 15 de abril de 2020 y fue respondido por docentes de 72 unidades curriculares que se estaban desarrollando en el semestre impar.

Es importante señalar las características que tuvo el inicio de la pandemia, que tomó de sorpresa a toda la población, y, específicamente para el caso de la enseñanza de nuestro servicio, únicamente se habían dictado dos semanas de clase, lo cual en muchos casos complejizó el contacto con los estudiantes. En ese contexto, las y los docentes se vieron inmersos en una situación que sin duda sobrepasó todas las previsiones para el dictado de las clases. 
A partir del formulario mencionado, encontramos casos de docentes que nunca habían tenido un curso en EVA, por lo cual sus primeras acciones fueron realizar las gestiones correspondientes para solicitar la creación del curso y del usuario, al tiempo de comenzar a familiarizarse con el entorno. Consultados/as acerca de los diferentes recursos utilizados para comunicarse con sus estudiantes, un $62,5 \%$ de los/as docentes afirmaron utilizar el correo electrónico. Un 58,3\% de los participantes de la encuesta utilizó los mensajes a través de la plataforma EVA, y un 19,4\% recurrió a los grupos de Whatsapp. Este último recurso, al igual que el correo electrónico, implicó que los docentes realizaran una solicitud a Bedelía para acceder al listado de inscriptos, para luego iniciar la comunicación.

Con relación al uso de la plataforma EVA, un 72.2\% de los docentes encuestados afirmó contar con un curso en EVA, mientras que otros estaban en ese momento gestionando la creación de uno, pero en otros casos hubo una explicitación de que no se consideraba usar la plataforma. En palabras del docente: "No tengo pensado trabajar con la plataforma EVA, como no lo he hecho en los últimos semestres". La dificultad que existía en ese momento, era que al haber transcurrido sólo dos semanas de clase, no todos los estudiantes estaban matriculados a los cursos en los que estaban inscriptos, lo cual requería de la complementariedad de esta vía de comunicación con otras alternativas.

Sobre el conocimiento de las herramientas que brinda la plataforma EVA, un $86,1 \%$ señaló conocerlas. Sin embargo, al profundizar acerca del uso concreto que se realizaba en ese momento de los diferentes recursos disponibles, un $45.8 \%$ de los docentes señaló que se utilizaba la plataforma como repositorio de materiales (bibliografía, PPTs, entre otros). Un 12.5\% de los encuestados afirmó utilizar los foros, y un $8.9 \%$ el recurso de BigBlueButton.

Consultados con relación a la posibilidad de utilizar otras herramientas para la enseñanza virtual, un 58.3\% de los docentes encuestados confirmó utilizarlas. Dentro de las mencionadas se destaca el correo electrónico con el $29.2 \%$ de las respuestas, y la plataforma ZOOM con el 9.7\%. También se mencionan, pero con porcentajes muy menores, Google Drive, Youtube, Whatsapp, Google Classroom, entre otros.

Específicamente en lo que refiere al desarrollo de clases en modalidad virtual, un $65.3 \%$ afirmó conocer las herramientas que harían posible el dictado de una clase de este tipo, pero solamente el $50 \%$ de los encuestados confirmó que tenía previsto en ese momento utilizarlas. Un 34.7\% dijo que tal vez las utilizaría, un $12.5 \%$ señaló que no las usaría, y un $2.8 \%$ no respondió a la pregunta. Dentro de las herramientas que los docentes pensaban utilizar al inicio de la pandemia, se destaca ZOOM, con el $31.9 \%$ de las respuestas, y BigBlueButton con el 30.6\%.

Por último, la encuesta intentó recoger las dificultades que estaban percibiendo los docentes en ese momento. Un 38.9\% de los encuestados afirmó tener dificultades en la utilización de las herramientas que había implementado. Algunas de ellas refirieron a la estabilidad de la plataforma EVA y problemas para la subida de archivos de gran tamaño; el uso del BigBlueButton; falta de infraestructura adecuada para el teletrabajo, dificultades de conectividad de parte de los estudiantes, entre otras. 
En este sentido, resulta necesario destacar la situación de ese momento, donde tanto estudiantes como docentes tuvieron que recurrir a las herramientas y posibilidades que tenían para continuar con los cursos. En este escenario, también Bedelía, Decanato, Medios técnicos y la Unidad de Apoyo a la Enseñanza vieron recargadas sus funciones y trataron de actuar en consecuencia, en una situación imprevista, y para la cual no había antecedentes, sumándose la migración a una nueva plataforma de correo institucional y del EVA FHCE al EVA Central. Es en este contexto en el que los/as docentes debieron compatibilizar sus tareas de enseñanza con la vida dentro del hogar, con la infraestructura y las herramientas que tenían disponibles, y en muchos casos con nuevas responsabilidades que atender en el marco de cuidados y apoyo familiar.

\section{Vamos avanzando}

Con la dinámica angustiosa del primer semestre del 2020, desde la UAE nos vimos en la necesidad de obtener información acerca del desarrollo de los cursos sin sobrecargar al personal docente con instrumentos, cuestionarios y planillas. Así fue como, con la idea de identificar los cursos que tuvieran mayor necesidad de acompañamiento, se elaboró una data de los cursos desarrollados en el semestre donde vertimos información obtenida de: la visita a los cursos en EVA FHCE y en EVA-UdelaR, y las informaciones compartidas a través de correos, salas virtuales de consulta, conversaciones telefónicas, videollamadas de whatsapp y demás medios de comunicación habilitados para asistir a los docentes.

Esta data da cuenta de 129 cursos observados en dos momentos: inicios del mes de abril y mediados del mes de mayo (abarcando 6 semanas de la evolución del semestre). Y en ella, a grandes rasgos, pudimos notar el siguiente estado de situación:

En el mes de abril, 23 cursos estaban muy bien desarrollados en EVA, con módulos completos, clases grabadas, variedad de recursos, explorando actividades de evaluación y evidencia de revisión y ajuste de contenidos y actividades según el contexto, con anuncios y mensajería activa con sus estudiantes.

25 cursos con buen grado de avance y 15 iniciando su configuración, lo que nos da un total de 63 cursos que estaban en desarrollo a travès de EVA con un evidente esfuerzo por parte de sus equipos docentes. (48,8\% del total de cursos sistematizados).

Por otra parte, 18 cursos estaban en una situación muy incipiente, apenas creados, con uno o dos materiales cargados, sin comunicación con estudiantes, o con material de años anteriores sin que fuera actualizado por sus docentes, lo que evidencia ausencia de actividad en el curso.

48 cursos no estaban en ninguna de los EVAs y de éstos sólo 11 reportaron estar utilizando otras plataformas de comunicación con sus estudiantes (correos, whatsapp, drive, blogs, hangouts, loom, entre otras). De todos ellos, 26 no reportaban contacto por ningún medio con sus estudiantes a esta fecha, siendo alarmante la situación dado el avance del semestre. Con ello, había 11 cursos de los que no se tenía ninguna información acerca del modo cómo estaban desarrollando sus actividades y qué soluciones habían encontrado. 
En el mes de mayo, luego de diversas intervenciones para atender los cursos con mayores dificultades, reuniones con institutos y comisiones, atención personalizada a docentes, observamos estas variaciones:

Se añade un curso más para un total de 24 cursos con desarrollo completo y ajustado al contexto, con variedad de recursos y actividades. Ya teníamos también 31 cursos con buen grado de avance, y 20 organizando su información, para un total de 75 cursos en desarrollo a travès de EVA, es decir, que 12 cursos avanzaron en su proceso con respecto al mes de abril.

13 cursos se mantenían con poco o nada, es decir que 5 cursos avanzaron de esta situación a mejorar su desarrollo.

Seguimos con cursos 41 sin EVA, con 12 reportando actividad mediante otras plataformas y/o herramientas virtuales. El resto de cursos de los que teníamos información difusa, correspondían a EFIS (13), seminarios y/o talleres fusionados (4), o cursos prácticos cuya compleja situación obligó a diferirlos o replantearlos.

Un detalle no menor es que en este período, docentes de 34 cursos distintos solicitaron asesoría y asistieron a instancias con la UAE para apoyarse en cuanto al desarrollo de sus cursos, notándose la incidencia de tales intervenciones en cuanto al manejo de la plataforma.

También es de hacer notar que muchos docentes eran de reciente ingreso a la FHCE, razón por la cual no habían creado usuario ni abierto sus cursos. En tales casos, 4 específicamente, se dio acompañamiento personalizado durante todo el proceso del semestre.

Este período también se caracterizó por una sobrecarga importante de trabajo en docentes que se vieron en la necesidad de prepararse aceleradamente en el uso de herramientas que les permitieran atender sus estudiantes, al tiempo que desarrollaban las actividades de sus cursos, atendían sus situaciones cotidianas y convivían con tareas de cuidado que se potenciaron con la cuarentena. Por ende, las atenciones y acompañamientos se orientaron a dar respuestas concretas y funcionales que les facilitaran el proceso con especial atención a aquellos docentes que usaban por primera vez la EVA, cualesquiera fueran las razones.

\section{La evaluación en tiempos de pandemia}

Al llegar al fin de mayo se hizo necesario recoger información acerca de las modalidades de evaluación que llevarían adelante los diversos cursos del semestre impar de la FHCE. Para ello, desde el Decanato y con la colaboración de la UAE, se solicitó a los equipos docentes que completaran una ficha donde se recogía información acerca de los equipos docentes, las modalidades de evaluación y las tareas de enseñanza virtual que se estaban desarrollando hasta el momento.

Del total de cursos que se dictaron en ese semestre, enviaron respuesta 102 de ellos, lo cual representa casi un $80 \%$. En relación con las evaluaciones, se observa de parte de los docentes la necesidad de adecuarlas a la modalidad virtual. Para el momento del relevamiento, casi todos los cursos ya tenían prevista la forma de evaluar, y en muchos de ellos también ya estaban fijadas las fechas. 
En cuanto a las modalidades propuestas, sigue primando la evaluación a través de parciales. Si bien en algunos casos los parciales se complementan con otras actividades, 53 de los 102 cursos aplicarían este tipo de pruebas. Hay 47 cursos que de acuerdo a lo previsto en mayo de 2020 preveían evaluar a través de trabajos domiciliarios, utilizando la modalidad de evaluación contínua a través de diferentes herramientas (producción de textos, análisis de videos, cuestionarios de múltiple opción, entre otros). Por último, 26 cursos plantean la existencia de un examen final, en algún caso obligatorio, y en otros como posibilidad para quienes no logran llegar a la calificación requerida a través de los parciales o trabajos domiciliarios.

En el marco de este relevamiento, los docentes comentan la necesidad que tuvieron de adecuar las modalidades de evaluación previstas en el programa del curso con la nueva situación de enseñanza virtual, al tiempo que fue necesario ajustar el cronograma previsto. Esto implicó generar nuevas estrategias, para lo cual se requirió una importante cantidad de horas de trabajo para familiarizarse con las herramientas informáticas y generar recursos y evaluaciones adecuadas a este nuevo contexto. Dicha situación fue sin dudas un desafío para toda la comunidad educativa, y en algunos casos ha provocado sentimientos de angustia y cansancio. En palabras de un docente, "Esta forma de trabajar es realmente agotadora (es difícil poner una pausa) y genera en mí mucha frustración y angustia. El esfuerzo es muy grande y los resultados no se condicen con las muchas horas invertidas. La dinámica que le he impuesto (...) requiere más horas de las que trabajo en la FHCE (20 horas semanales), situación del todo irregular".

Se ha perdido el control de las horas de trabajo, en este contexto de intercalar constantemente la vida doméstica y familiar con la vida laboral. Los tiempos para preparar clases y materiales son además mucho mayores que en tiempos de "normalidad". Según un docente, "Además del tiempo que insume la planificación y el dictado de un curso en esta modalidad, la situación de emergencia social ha llevado a considerar aspectos vinculados con la vida no académica tanto nuestra como de los estudiantes con una dedicación no medible en términos de horas".

\section{Reflexiones finales}

El advenimiento de la pandemia del COVID-19 tuvo consecuencias en todas las esferas de la sociedad. En el caso de la educación superior, y de la FHCE en particular, implicó un gran esfuerzo de diversas unidades para tomar resoluciones rápidas y facilitar el pasaje de la enseñanza presencial a la virtual. Bedelía, Decanato, Medios Técnicos y la UAE debieron trabajar de forma coordinada, y generando acciones concretas para apoyar tanto a estudiantes como docentes. Estos últimos, tuvieron que adaptar sus cursos a la nueva realidad, familiarizarse con herramientas de enseñanza virtual que en muchos casos nunca habían utilizado, y compatibilizar su labor docente con las responsabilidades domésticas y de cuidado que se vieron potenciadas con la pandemia. Este proceso llevó a los docentes a reflexionar sobre sus propias prácticas, adecuar su planificación a las posibilidades con las que contaban desde sus hogares, modificando y adaptándose a las nuevas situaciones que se observaban a partir de las prácticas. 
En cada uno de los "momentos" mostrados en esta sistematización, quedan en evidencia elementos acerca de los cuales hay que seguir trabajando de cara a una real transformación de prácticas. Problematizar aspectos como la calidad educativa, la dotación de infraestructuras informáticas y de conectividad, la figura del docente, el estudiante como sujeto de la enseñanza, la prosecución académica, la deserción, la inclusión educativa (de personas con capacidades diversas, de mujeres que atienden roles de cuidado, de personas que viven en el interior del país, de quienes no tienen acceso a recursos informáticos, entre otras condiciones), son apenas algunos de los temas que quedan aún por debatir a partir de las experiencias concretas de cada institución universitaria.

En todos los casos, queda interpelada la universidad, el sistema universitario y el modo cómo cada docente asume su rol, evaluando cuáles aspectos deben continuar y cuáles deben transformarse, todo ello en función del modelo de universidad que se espera construir. He allí el gran debate aún por hacer.

La información presentada en este trabajo surgió en el marco del rápido accionar para atender la situación de emergencia, obteniendo datos parciales, de aquellos cursos que respondieron a la convocatoria, y en todos los casos generados para tomar decisiones. Consideramos que este es un primer avance para dar cuenta de lo acontecido en este período, pero que se hace necesario profundizar en la generación de investigaciones con herramientas específicas para el análisis y reflexión. Creemos que es una oportunidad para repensarnos y ajustar nuestras prácticas hacia nuevos horizontes.

\section{Bibliografía}

Anijovich, R. y Cappelletti, G. (2017). La evaluación como oportunidad. Buenos Aires: Paidós.

Álvarez, Z., Porta, L. y Sarasa, M.C. (2010). La investigación narrativa en la enseñanza: las buenas prácticas y las biografías de los profesores memorables. Revista de Educación, 1(1), 159-179.

Barnechea, M.M., González, E. y Morgan, M. (1999). La producción de conocimientos en sistematización. La Piragua. Revista latinoamericana de educación y política, 16, 33-43

Bates, A. T. (2018). La Enseñanza en la era digital una guía para la enseñanza y el aprendizaje. Recuperado de https://cead.pressbooks.com/

Borjas, B. (2003). Metodología para sistematizar prácticas educativas: Por las ciudades de ítalo Calvino. Caracas: Federación Internacional de Fe y Alegría

Castañeda, L., Esteve, F. y Adell, J. (2018). ¿Por qué es necesario repensar la competencia docente para el mundo digital? Revista de Educación a Distancia, 56. http://dx.doi.org/10.6018/ red/56/6

Cobo, C. (2019). Acepto las condiciones. Usos y abusos de las tecnologías digitales. Madrid: Fundación Santillana.

Coppens, F. y Van de Velde, H. (2005). Sistematización. Texto de referencia y de consulta. Módulo 6 - Curso E-Dc-6.1 Nicaragua: CURN/CICAP

Dussel, I., Ferrante, P. y Pulfer, D. (Comps.) (2020). Pensar la educación en tiempos de pandemia: entre la emergencia, el compromiso y la espera. Buenos Aires: UNIPE/CLACSO

Dron, J. (2018). Smart learning environments, and not so smart learning environments: a systems view. Smart Learning Environments, 5(1), 1-20. https://doi.org/10.1186/s40561-018-0075-9 
Fenstermacher, E. (1989). Tres aspectos de la investigación en la enseñanza, en: Wittrork, M. La investigación de la enseñanza. Enfoques, teorías y métodos. Barcelona: Paidós.

González-Sanmamed, M., Sangrà, A., Souto-Seijo, A. y Estévez, I. (2018). Ecologías de aprendizaje en la Era Digital: desafíos para la Educación Superior. Publicaciones, 48(1), 25-45. https:// doi.org/10.30827/publicaciones.v48i1.7329

Hidalgo, A., León, G. y Pavón, J. (2002). La gestión de la innovación y la tecnología en las organizaciones. Madrid: Pirámide.

Jara, O. (1994). Para sistematizar experiencias. Costa Rica: Alforja

Lion, C. (2017). Tecnologías y aprendizajes: claves para repensar la escuela. En: Montes. N. (Comp.) Educación y TIC. De las políticas a las aulas. Buenos Aires: Eudeba

Martel, A. (2004). La educación social e individual en la era de la educación a distancia en la globalización. Revista Electrónica de Investigación y Desarrollo Educativo. Vol 6. Universidad Autónoma de Baja California, México. Disponible en https://redie.uabc.mx/redie/article/view/97

Morales, N. (2019). La incorporación de la competencia digital docente en estudiantes y docentes de formación inicial docente en Uruguay para personas con parálisis cerebral. [Tesis de doctorado, Universitat Rovira I Virgili]. Disponible en https://www.tdx.cat/ handle/10803/667661

Polanía, J. (2014). Blended Learning, una alternativa para E-learning:n modelo Educativo. Paideia Surcolombiana, 2(19), 91-96. https://doi.org/10.25054/01240307.1170

Porta, M. y Rodés, V (2020) Metodologías para el rediseño de la enseñanza en ambientes digitales. Programa de Entornos Virtuales de Aprendizaje (ProEVA) Departamento de Apoyo Técnico Académico (DATA) Universidad de la República, Uruguay. Recuperado de https:// bit.ly/36Rr1P9

Porto, D. (2012). Narrativas Transmedia. Entre teorías y prácticas. Bogotá: Editorial Universidad del Rosario

Rodríguez, C., Czerwonogora, A., Verde, J. y Doninalli, M. (2014). Generando la trama transversal: actividades de evaluación formativa en la virtualidad. En: C. Rodríguez, A. Czerwonogora, J. Verde \& M. Doninalli. Evaluación formativa y herramientas tecnológicas. Aportes transversales más allá de las aulas. Montevideo: UdelaR, Ediciones Universitarias. Recuperado de https://bit.ly/3wStUd7

Rodríguez, C. y Rodés, V (2020) Métodos y herramientas para la evaluación en línea de los aprendizajes. Programa de Entornos Virtuales de Aprendizaje (ProEVA) Departamento de Apoyo Técnico Académico (DATA) Universidad de la República, Uruguay. Recuperado de https://bit.ly/3wXFS59

Scolari, C (2018). Alfabetismo transmedia en la nueva ecología de los medios. Barcelona: H2020 TRANSLITERACY Project. Recuperado de https://bit.ly/37A4ITT

Vera, F. (2008). La modalidad Blended-Learning en la educación superior. Recuperado de https:// bit.ly/3eLqQJw

Vólquez, J. A. y Amador, C. M. (2020). Competencias digitales de docentes de nivel secundario de Santo Domingo: un estudio de caso. RIDE Revista Iberoamericana Para La Investigación y el Desarrollo Educativo, 11(21). https://doi.org/10.23913/ride.v11i21.702 